SUPPORTING INFORMATION

\title{
4-Aryl-1,2,3-triazole; A Novel Template for a Reversible Methionine Aminopeptidase 2 Inhibitor, Optimized to Inhibit Angiogenesis in vivo
}

Lara S. Kallander, ${ }^{*}$ Qing Lu, Wenfang Chen, Thaddeus Tomaszek, Guang Yang, David Tew, Thomas D. Meek, Glenn A. Hofmann, Christina K. Schulz-Pritchard, Ward. W. Smith, Cheryl A. J anson, M. Dominic Ryan, Gui-feng Zhang, Kyung O. J ohanson, Robert B. Kirkpatrick, Thau F. Ho, Paul W. Fisher, Michael R. Mattern, Randall K. J ohnson, Michael J . Hansbury, J ames D. Winkler, Keith W. Ward, Daniel F. Veber, and Scott K. Thompson

\section{Table of Contents}

X-ray Crystal Structure Determination and Refinement Statistics $\quad$ S1

Enzyme Inhibition $\quad S 1$

Cellular Inhibition $\quad$ S2

Pharmacokinetics $\quad$ S2

Matrigel Angiogenesis Model $\quad$ S3

$\begin{array}{ll}\text { Chemistry } & \text { S3 }\end{array}$

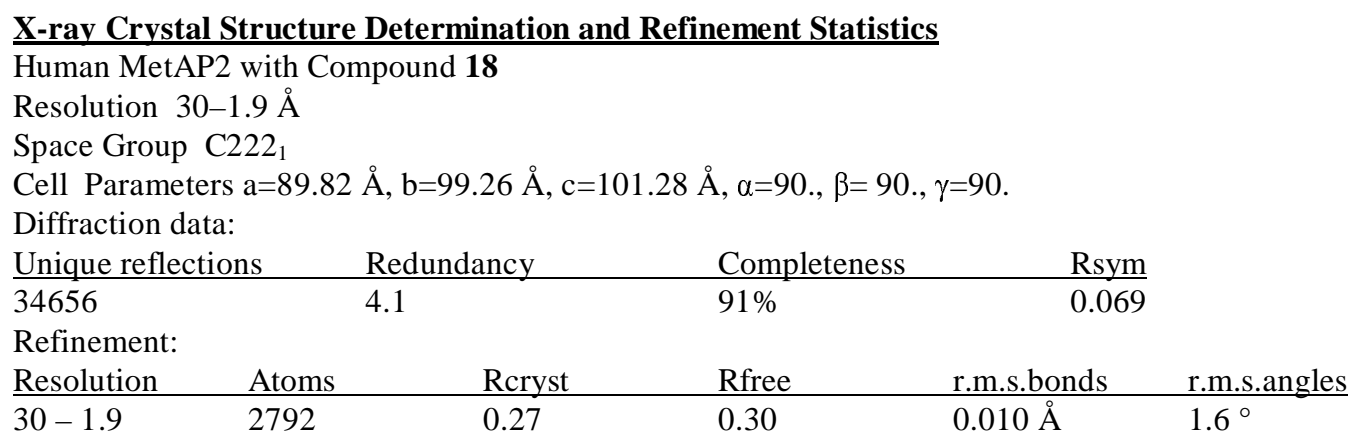

\section{Enzyme Inhibition:}

Human methionine aminopeptidase 2 was expressed and purified as described previously ${ }^{1}$ in the presence of $\mathrm{Co}^{+2}$. HMetAP2 activity was monitored by measuring the initial velocity of turnover of the artificial substrate L-methionine-7-amido-4-methylcoumarin (Met-AMC). Assays were performed in 96 well microtitre plates using recombinant $\mathrm{N}$-terminal truncated human methionine aminopeptidase type II with $\mathrm{Co}^{2+}$ as a cofactor. In a typical 96-well plate assay, the increase in the fluorescence emission $\left(\lambda_{\mathrm{ex}}=360 \mathrm{~nm}, \lambda_{\mathrm{em}}=460 \mathrm{~nm}\right.$ ) of a $50 \mu \mathrm{L}$ assay solution in each well was monitored continuously using a Cytofluor series 4000 multiwell plate reader (PerSeptive Biosystems) and used to calculate the initial velocity of hMetAP2. Each $50 \mu \mathrm{L}$ assay solution, contained $50 \mathrm{mM}$ Hepes $\cdot \mathrm{Na}^{+}$( $\mathrm{pH} 7.5$ ), $100 \mathrm{mM} \mathrm{NaCl}, 10 \mathrm{nM}$ purified hMetAP2 enzyme, and 0.4 $\mathrm{mM}$ Met-AMC (in $1 \%$ DMSO aqueous solution, $\mathrm{K}_{\mathrm{m}}=0.3 \mathrm{mM}$ with $\mathrm{Co}^{+2}$ as a cofactor). Assays were initiated with the addition of substrate and the initial rates were corrected for the background rate determined in the absence of hMetAP2. Data were fitted to the Dixon competitive inhibition equation using Grafit (Erithacus Software).

\footnotetext{
${ }^{1}$ Yang, G.; Kirkpatrick, R. B.; Ho, T.; Zhang, G-F.; Liang, P-H.; Johanson, K. O.; Casper, D. J.; Doyle, M. L.; Marino, J. P., Jr.; Thompson, S. K.; Chen, W.; Tew, D. G.; Meek, T. D. Steady-State Kinetic Characterization of Substrates and Metal-Ion Specificities of the Full-Length and N-Terminally Truncated Recombinant Human Methionine Aminopeptidases (Type 2). Biochem. 2001, 40, 1064510654.
} 
For use in the manganese activation assay, the cobalt metal was removed from MetAP2 by treatment with EDTA. ${ }^{2}$ HMetAP2 activity was monitored by measuring the initial velocity of turnover of the artificial substrate L-methionine-7-amido-4-trifluoromethylcoumarin (Met-AFC). Assays were performed in 96 well microtitre plates using recombinant N-terminal truncated human methionine aminopeptidase type II with $25 \mu \mathrm{M} \mathrm{Mn}^{+2}$ added as a cofactor. In a typical 96well plate assay, the increase in the fluorescence emission $\left(\lambda_{\mathrm{ex}}=405 \mathrm{~nm}, \lambda_{\mathrm{em}}=530 \mathrm{~nm}\right)$ of a $50 \mu \mathrm{L}$ assay solution in each well was monitored and used to calculate the initial velocity of hMetAP2. Each $50 \mu \mathrm{L}$ assay solution, contained $50 \mathrm{mM}$ Hepes. $\mathrm{Na}^{+}$(pH 7.5), $100 \mathrm{mM} \mathrm{NaCl}, 23 \mathrm{nM}$ purified hMetAP2 enzyme, and $2 \mathrm{mM}$ Met-AFC (in $1 \%$ DMSO aqueous solution, $\mathrm{K}_{\mathrm{m}}=5 \mathrm{mM}$ with $\mathrm{Mn}^{+2}$ as a cofactor).

\section{Cellular Inhibition:}

XTT $72 \mathrm{hr}$ growth inhibition protocol for mammalian cultured cells

Nonendothelial human lines were grown in Dulbecco's modified Eagle's medium (DMEM) also containing 10\% fetal bovine serum (FBS) and the antibiotics penicillin (100 Units/ml) and streptomycin (100 micrograms/ml) (Invitrogen / Life Technologies). HUVECs were obtained from VEC Technologies (Rensselaer, NY) and grown in MCDB-131 complete endothelial cell culture medium obtained from this supplier. Cells were grown in $37^{\circ} \mathrm{C}$ in humidified $5 \% \mathrm{CO}_{2}$ incubators in $75 \mathrm{~cm}^{2}$ plastic flasks. They were harvested using $0.25 \%$ trypsin / $1 \mathrm{mM}$ ethylenediamine-tetraacetic acid (EDTA), resuspended in growth medium, and counted using a hemocytometer. Flat-bottomed 96-well plates were seeded with $2-4 \times 10^{3}$ cells/well in a volume of $200 \mathrm{ul}$ from trypsinized exponentially growing cultures. To "blank" wells, growth medium was added with no additions. Cells were incubated overnight to permit attachment.

Next day, medium from wells that contained cells was replaced with 180 microliters of fresh medium. Appropriate dilutions of test compounds were added to the wells from stock soloutions of compound dissolved in dimethyl sulfoxide (DMSO); final DMSO concentration in all wells was $0.2 \%$. Cells plus compound were incubated for an additional $72 \mathrm{hr}$ at $37^{\circ} \mathrm{C}$ under normal growth conditions. Cells were then assayed for viability using standard XTT/PMS (described below). Fifty microliters of XTT/PMS solution was added to each well and plates were incubated for 90 minutes at $37^{\circ} \mathrm{C}$. Absorbance at $450 \mathrm{nM}$ was then determined using a 96-well UV plate reader (Molecular Devices). Under these conditions, absorbance of untreated control cells at $450 \mathrm{~nm}$ was at least 1.0 optical density unit/ml. Percent viability of cells in each well was calculated from these data (having been corrected for background absorbance). It was equal to

$100 \times$ (A450 test well / A450 untreated control well),

the $\mathrm{A} 450 \mathrm{~s}$ being averages of triplicate determinations. $\mathrm{IC}_{50}$ was that concentration of compound that reduced cell viability to $50 \%$ of control (untreated) viability, as determined from plots of concentration vs percent viability.

-Preparation of XTT/PMS solution (immediately before assay).

For each 96-well plate, 8 mg XTT (2,3-bis[2-Methoxy-4-nitro-5-sulfophenyl]-2H-tetrazolium-5carboxanilide) (Sigma Chemical Co.) per plate was dissolved in $100 \mathrm{ul}$ DMSO. $3.9 \mathrm{ml} \mathrm{H}_{2} \mathrm{O}$ was added to dissolve XTT and $20 \mathrm{ul}$ of PMS (phenazine methosulfate, Sigma Chemical Co.) stock solution $(3.0 \mathrm{mg} / \mathrm{ml})$ was added from frozen aliquoted stock solution $(10 \mathrm{mg}$ of PMS in $3.3 \mathrm{ml}$ phosphate buffered saline (Invitrogen / Life Technologies). (These stocks are routinely frozen at $20^{\circ} \mathrm{C}$ until use).

\section{Pharmacokinetics:}

Mouse oral exposure was determined in 3 female C57BL/6 mice. Each animal (fed) received Compound 24 as an oral gavage (160 umol/kg target dose; $16 \mathrm{~mL} / \mathrm{kg}$ dose volume) formulated as a solution in $20 \%$ aqueous hydroxypropyl-beta-cyclodextrin ( $\mathrm{pH} 4.0)$ with $1 \%$ DMSO. Blood samples were collected from a lateral tail vein at various times following oral dosing, and a $25-\mathrm{uL}$ blood aliquot was added to $25 \mathrm{uL}$ of water and allowed to stand on ice for approximately 5 min to

\footnotetext{
2 Walker, K. W.; Bradshaw, R. A. Yeast Methionine Aminopeptidase 1 Can Utilize Either $\mathrm{Zn}+2$ or $\mathrm{Co}+2$ as a Cofactor: A Case of Mistaken Identity? Protein Sci., 1998, 7, 2684-2687.
} 
facilitate hemolysis. Rat pharmacokinetics were determined in 3 male Sprague-Dawley rats in a crossover fashion. On study day 1, each animal (fed) received Compound 24 as a 30-min intravenous infusion ( $8 \mathrm{umol} / \mathrm{kg}$ target dose; $4 \mathrm{~mL} / \mathrm{kg}$ dose volume) formulated as a solution in $20 \%$ aqueous hydroxypropyl-beta-cyclodextrin ( $\mathrm{pH} 3.5$ ) with $1.5 \%$ DMSO. On study day 2, the same animals (fasted) received Compound $\mathbf{2 4}$ as an oral gavage (16 umol/kg target dose; 16 $\mathrm{mL} / \mathrm{kg}$ dose volume) formulated as a solution in $6 \%$ aqueous hydroxypropyl-beta-cyclodextrin ( $\mathrm{pH} 4.5$ ) with $0.7 \%$ DMSO. Blood samples were collected from a lateral tail vein at various times following dosing and centrifuged to obtain plasma. Blood and plasma concentrations of Compound 24 from the rat and mouse experiments were quantified by LC/MS/MS (LLQ = 10 $\mathrm{ng} / \mathrm{mL}$ for both matrices). Noncompartmental methods were used for analysis of concentration versus time data. DNAUC - dose-normalized area under the concentration-time curve.

\section{Matrigel Angiogenesis Model:}

All in-vivo experiments were carried out in accord with protocols approved by the GSK IACUC. Basic FGF (bFGF-R\&D Systems, Minneapolis, MN.) was added as an angiogenic stimulant, to a final concentration of 500ng/ $\mathrm{ml}$ to reduced growth factor, endotoxin free Matrigel (Becton-Dickinson, Bedford, Massachusetts). Eight week old C57 female mice were administered $0.5 \mathrm{ml}$ of the matrigel by dorsal subcutaneous injection through chilled syringes. At physiological temperature, the liquid matrigel rapidly forms a solid and cohesive gel.

Over the course of the next six days, the mice were dosed by oral gavage three times daily, with compound formulated in a $20 \%$ solution of encapsin. After 6 days of treatment, the mice were scarificed and the matrigel plugs recovered and homogenized in saline. ${ }^{3}$ Angiogenesis was quantitated by analyzing the hemoglobin content of the gel by a colorimetric assay (Plasma Hemoglobin-Sigma) ${ }^{4}$. Data were analyzed for variance and then for significance by paired, two-tailed, t-test versus control.

\section{Chemistry:}

In the examples, materials were obtained from commercial suppliers and used without further purification. Preparative HPLC purification was performed on a Gilson HPLC with a YMC CombiPrep ODS-A using a gradient of acetonitrile:water as elutant and UV detection at $214 \mathrm{~nm}$. Kieselgel 60 silica gel was used for chromatography. Proton NMR spectra were performed on a Bruker $400 \mathrm{MHz}$ NMR spectrometer. Mass spectra were taken on a PE Sciex API-150 instrument using electrospray ionization (ESI). Elemental analysis was performed by Quantitative Technologies Inc.

(5) 2,8-dihydro-indeno[1,2-d]-1,2,3-triazole was a gift from Henry Rapoport ${ }^{5}$

Representative procedure for the preparation of phenylalkynes 6 : To a stirring solution of 2methylbenzaldehyde $(0.35 \mathrm{~mL}, 3.0 \mathrm{mmol})$ in dry methanol $(30 \mathrm{~mL})$ was added potassium carbonate $(0.87 \mathrm{~g}, 6.3 \mathrm{mmol})$ and 1-diazo-2-oxopropylphosphonate $(0.77 \mathrm{~g}, 4.0 \mathrm{mmol}){ }^{7}$. After $4 \mathrm{~h}$ of stirring at room temperature, aqueous sodium bicarbonate $(5 \%, 50 \mathrm{~mL})$ and hexanes $(50 \mathrm{~mL})$ were added. The organic layer was collected, dried $\left(\mathrm{MgSO}_{4}\right)$ and filtered through a short silica plug. Evaporation gave 1-ethynyl-2-methylbenzene as an oil, 93\% yield, ${ }^{1} \mathrm{H}-\mathrm{NMR}(400 \mathrm{MHz}$, $\left.\mathrm{CDCl}_{3}\right): \delta 7.48(\mathrm{~d}, J=7.5 \mathrm{~Hz}, 1 \mathrm{H}), 7.16-7.27(\mathrm{~m}, 3 \mathrm{H}), 3.29(\mathrm{~s}, 1 \mathrm{H}), 2.47(\mathrm{~s}, 3 \mathrm{H})$.

1-ethynyl-2-methoxybenzene Oil, 26\% yield, ${ }^{1} \mathrm{H}-\mathrm{NMR}\left(400 \mathrm{MHz}, \mathrm{CDCl}_{3}\right): \delta 7.48(\mathrm{~m}, 1 \mathrm{H}), 7.35(\mathrm{~m}, 1 \mathrm{H}), 6.92(\mathrm{~m}, 2 \mathrm{H}), 3.93(\mathrm{~s}$, $3 \mathrm{H}), 3.34$ (s, 1H).

\section{1-ethynyl-4-iodobenzene}

White solid, 71\% yield, ${ }^{1} \mathrm{H}-\mathrm{NMR}\left(400 \mathrm{MHz}, \mathrm{CDCl}_{3}\right): \delta 7.68(\mathrm{~d}, J=8.3 \mathrm{~Hz}, 2 \mathrm{H}), 7.23(\mathrm{~d}, J=8.3$ $\mathrm{Hz}, 2 \mathrm{H}), 3.17$ (s, 1H).

\footnotetext{
3 Passaniti, A.; Taylor, R. M.; Pili, R.; Guo, Y.; Long, P. V.; Haney, J. A.; Pauly, R. R.; Grant, D. S.; Martin, G. R. A simple, quantitative method for assessing angiogenesis and antiangiogenic agents using reconstituted basement membrane, heparin, and fibroblast growth factor. Lab. Invest. 1992, 67, 519-528.

${ }^{4}$ Lijana, R.C.; Williams, M.C. Tetramethylbenzidine - a Substitute for Benzidine in Hemoglobin Analysis. J. Lab. Clin. Med., 1979, 94, 266-276.

5 Rapoport, H.; Chen, H. H. Wolff-Kishner reaction with $\alpha$-hydroxyimino ketones. J. Org. Chem. 1960, $25,313$.

6 Mueller, S.; Liepold, B.; Roth, G. J.; Bestmann, H. J. An Improved One-pot Procedure for the Synthesis of Alkynes from Aldehydes. Synlett 1996, 6, 521-522.

${ }^{7}$ Callant, P.; D'Haenens, L.; Van der Eycken, E.; Vandewalle, M.. Photoinduced Wolff rearrangement of $\alpha$-diazo- $\beta$-ketophosphonates: a novel entry into substituted phosphonoacetates. Synth. Comm. 1984, 14, 163-167.
} 
1-bromo-3-ethynylbenzene

Oil, $84 \%$ yield, ${ }^{1} \mathrm{H}-\mathrm{NMR}\left(400 \mathrm{MHz}, \mathrm{CDCl}_{3}\right): \delta 7.66(\mathrm{~s}, 1 \mathrm{H}), 7.50(\mathrm{~d}, J=8.0 \mathrm{~Hz}, 1 \mathrm{H}), 7.44(\mathrm{~d}, J=$ $7.7 \mathrm{~Hz}, 1 \mathrm{H}), 7.21(\mathrm{t}, J=7.9 \mathrm{~Hz}, 1 \mathrm{H}), 3.14(\mathrm{~s}, 1 \mathrm{H})$.

1-bromo-4-ethynylbenzene

White solid, 79\% yield, ${ }^{1} \mathrm{H}-\mathrm{NMR}\left(400 \mathrm{MHz}, \mathrm{CDCl}_{3}\right): \delta 7.48(\mathrm{~d}, J=8.5 \mathrm{~Hz}, 2 \mathrm{H}), 7.37(\mathrm{~d}, J=8.4$ $\mathrm{Hz}, 2 \mathrm{H}), 3.14$ (s, 1H).

1,2-dibromo-4-ethynylbenzene

White solid, $81 \%$ yield, ${ }^{1} \mathrm{H}-\mathrm{NMR}\left(400 \mathrm{MHz} \mathrm{CDCl}_{3}\right): \delta 7.64(\mathrm{~s}, 1 \mathrm{H}), 7.46(\mathrm{~d}, J=8.2 \mathrm{~Hz}, 1 \mathrm{H}), 7.16$ (d, $J=8.2 \mathrm{~Hz}, 1 \mathrm{H}), 3.07$ (s, 1H).

2-ethynylbiphenyl

Oil, $92 \%$ yield, ${ }^{1} \mathrm{H}-\mathrm{NMR}\left(400 \mathrm{MHz}, \mathrm{CDCl}_{3}\right): \delta$ 7.65-7.60 (m, 3H), 7.31-7.47 (m, 6H), $3.06(\mathrm{~s}$,

$1 \mathrm{H})$.

2-ethynyl-1-benzofuran

Oil, $85 \%$ yield, ${ }^{1} \mathrm{H}-\mathrm{NMR}\left(400 \mathrm{MHz}, \mathrm{CDCl}_{3}\right): \delta 7.59(\mathrm{~d}, J=7.8 \mathrm{~Hz}, 1 \mathrm{H}), 7.48(\mathrm{~d}, J=8.2 \mathrm{~Hz}, 1 \mathrm{H})$,

7.35-7.40 (m, 1H), 7.26-7.30 (m, 1H), $7.03(\mathrm{~s}, 1 \mathrm{H}), 3.52(\mathrm{~s}, 1 \mathrm{H})$.

3-ethynylpyridine

Oil, $81 \%$ yield, ${ }^{1} \mathrm{H}-\mathrm{NMR}\left(400 \mathrm{MHz}, \mathrm{CDCl}_{3}\right): \delta 8.74(\mathrm{~s}, 1 \mathrm{H}), 8.59(\mathrm{~d}, J=4.9 \mathrm{~Hz}, 1 \mathrm{H}), 7.80(\mathrm{~m}$,

$1 \mathrm{H}), 7.29(\mathrm{~m}, 1 \mathrm{H}), 3.24(\mathrm{~s}, 1 \mathrm{H})$.

4-ethynylpyridine

Brown solid, 85\% yield, ${ }^{1} \mathrm{H}-\mathrm{NMR}\left(400 \mathrm{MHz}, \mathrm{CDCl}_{3}\right): \delta 8.61(\mathrm{~d}, J=4.5 \mathrm{~Hz}, 2 \mathrm{H}), 7.37(\mathrm{~d}, J=4.5$

$\mathrm{Hz}, 2 \mathrm{H}), 3.31(\mathrm{~s}, 1 \mathrm{H})$.

2-ethynyl-6-methyl-pyridine

Oil, $99 \%$ yield, ${ }^{1} \mathrm{H}-\mathrm{NMR}\left(400 \mathrm{MHz}, \mathrm{CDCl}_{3}\right): \delta 7.56(\mathrm{t}, J=7.7 \mathrm{~Hz}, 1 \mathrm{H}), 7.32(\mathrm{~d}, J=7.7 \mathrm{~Hz}, 1 \mathrm{H})$,

$7.14(\mathrm{~d}, J=7.8 \mathrm{~Hz}, 1 \mathrm{H}), 3.14$ (s, 1H), 2.58 (s, 3H).

2-ethynyl-3-methylpyridine, 2-ethynyl-4-methylpyridine, and 2-ethynyl-5-methylpyridine were prepared as previously reported. 8

Representative procedure for the preparation of phenyl-1,2,3-triazoles ${ }^{9}$ : Commercially available or synthetically prepared ethynyl substrate were reacted in the following manner. To a stirring solution of 4-ethynyltoluene $(0.92 \mathrm{~g}, 7.9 \mathrm{mmol})$ in toluene $(8 \mathrm{~mL})$ under an inert atmosphere was added trimethylsilylazide $(2 \mathrm{~mL}, 15.0 \mathrm{mmol})$. The resulting solution was heated to reflux for 2 days. To this mixture was added water $(1.5 \mathrm{~mL})$ and after evaporation, the resulting residue was purified by reverse phase preparative HPLC to give 4-(4-methylphenyl)-1H-1,2,3-triazole (3) as a white solid, $14 \%$ yield, ${ }^{1} \mathrm{H}-\mathrm{NMR}\left(400 \mathrm{MHz}, \mathrm{CDCl}_{3}\right): \delta 11.58$ (br s, $\left.1 \mathrm{H}\right), 7.96(\mathrm{~s}, 1 \mathrm{H}), 7.73(\mathrm{~d}, J$ $=8.0 \mathrm{~Hz}, 2 \mathrm{H}), 7.28(\mathrm{~m}, 2 \mathrm{H}), 2.42(\mathrm{~s}, 3 \mathrm{H})$. MS (ESI) $160(\mathrm{M}+\mathrm{H})^{+}$.

(8) 4-(2-methylphenyl)-1H-1,2,3-triazole

White solid, 3\% yield, ${ }^{1} \mathrm{H}-\mathrm{NMR}\left(400 \mathrm{MHz}, \mathrm{CD}_{3} \mathrm{OD}\right): \delta 7.97(\mathrm{~s}, 1 \mathrm{H}), 7.56(\mathrm{~m}, 1 \mathrm{H}), 7.26-7.33(\mathrm{~m}$, $3 \mathrm{H}), 2.44$ (s, 3H). MS (ESI) $160(\mathrm{M}+\mathrm{H})^{+}$.

(9) 4-(2-methoxyphenyl)-1H-1,2,3-triazole

White solid, $12 \%$ yield, ${ }^{1} \mathrm{H}-\mathrm{NMR}\left(400 \mathrm{MHz}, \mathrm{CD}_{3} \mathrm{OD}\right): \delta 8.19(\mathrm{~s}, 1 \mathrm{H}), 7.95(\mathrm{~d}, J=6.8 \mathrm{~Hz}, 1 \mathrm{H})$, 7.35-7.40 (m, 1H), $7.14(\mathrm{~d}, J=8.3 \mathrm{z}, 1 \mathrm{H}), 7.04-7.08(\mathrm{~m}, 1 \mathrm{H}), 4.90(\mathrm{~s}, 3 \mathrm{H})$. MS (ESI) 176

$(\mathrm{M}+\mathrm{H})^{+}$.

(10) 4-(1-biphenyl-2-yl)-1H-1,2,3-triazole

Oil, $29 \%$ yield, ${ }^{1} \mathrm{H}-\mathrm{NMR}\left(400 \mathrm{MHz}, \mathrm{CD}_{3} \mathrm{OD}\right): \delta 7.80(\mathrm{~s}, 1 \mathrm{H}), 7.47-7.49(\mathrm{~m}, 2 \mathrm{H}), 7.36-7.40(\mathrm{~m}, 4$ H), 7.20-7.22 (m, 2H), 6.88 (s, 1H). MS (ESI) $222(\mathrm{M}+\mathrm{H})^{+}$.

(11) 4-(4-ethylphenyl)-1H-1,2,3-triazole

White solid, $11 \%$ yield, ${ }^{1} \mathrm{H}-\mathrm{NMR}\left(400 \mathrm{MHz}, \mathrm{CD}_{3} \mathrm{OD}\right): \delta 8.11(\mathrm{~s}, 1 \mathrm{H}), 7.74(\mathrm{~d}, J=8.2 \mathrm{~Hz}, 2 \mathrm{H})$, $7.30(\mathrm{~d}, J=8.2 \mathrm{~Hz}, 2 \mathrm{H}), 2.69(\mathrm{q}, J=7.6 \mathrm{~Hz}, 2 \mathrm{H}), 1.27(\mathrm{t}, J=7.6 \mathrm{~Hz}, 3 \mathrm{H})$. MS (ESI) $174(\mathrm{M}+\mathrm{H})^{+}$. (12) 4-(4-n-propylphenyl)-1H-1,2,3-triazole

White solid, $26 \%$ yield, ${ }^{1} \mathrm{H}-\mathrm{NMR}\left(400 \mathrm{MHz}, \mathrm{CD}_{3} \mathrm{OD}\right): \delta 8.11(\mathrm{~s}, 1 \mathrm{H}), 7.74(\mathrm{~d}, J=7.5 \mathrm{~Hz}, 2 \mathrm{H})$, $7.28(\mathrm{~d}, J=8.0 \mathrm{~Hz}, 2 \mathrm{H}), 2.64(\mathrm{t}, J=7.6 \mathrm{~Hz}, 2 \mathrm{H}), 1.64-1.73(\mathrm{~m}, 2 \mathrm{H}), 0.97(\mathrm{t}, J=7.3 \mathrm{~Hz}, 3 \mathrm{H}) . \mathrm{MS}$ (ESI) $188(\mathrm{M}+\mathrm{H})^{+}$.

(14) 4-(1H-1,2,3-triazol-4-yl)-phenylamine

\footnotetext{
8 Tsuchiya, T.; Kato, M.; Sashida, H. Thermal intramolecular cyclization of 2-ethynylpyridine N-ylides to indolizines and cyclazines. Chem. Pharm. Bull. 1984, 32, 4666-4669.

9 Tanaka, Y.; Velen, S. R.; Miller, S. I. Syntheses and Properties of 1,2,3-Triazoles. Tetrahedron 1973, 29, $3271-83$.
} 
Orange solid, $9 \%$ yield, ${ }^{1} \mathrm{H}-\mathrm{NMR}\left(400 \mathrm{MHz}, \mathrm{CD}_{3} \mathrm{OD}\right): \delta 7.94(\mathrm{~s}, 1 \mathrm{H}), 7.54(\mathrm{~d}, J=8.6 \mathrm{~Hz}, 2 \mathrm{H})$, $6.78(\mathrm{~d}, J=8.6 \mathrm{~Hz}, 2 \mathrm{H})$. MS (ESI) $161(\mathrm{M}+\mathrm{H})^{+}$.

(15) 4-(4-chlorophenyl)-1H-1,2,3-triazole

White solid, $35 \%$ yield, ${ }^{1} \mathrm{H}-\mathrm{NMR}\left(400 \mathrm{MHz}, \mathrm{CD}_{3} \mathrm{OD}\right): \delta 8.18(\mathrm{~s}, 1 \mathrm{H}), 7.85(\mathrm{~d}, J=8.6 \mathrm{~Hz}, 2 \mathrm{H})$, $7.47(\mathrm{~d}, J=8.7 \mathrm{~Hz}, 2 \mathrm{H})$. MS (ESI) $180(\mathrm{M}+\mathrm{H})^{+}$.

(16) 4-(4-bromophenyl)-1H-1,2,3-triazole

White solid, $21 \%$ yield, ${ }^{1} \mathrm{H}-\mathrm{NMR}\left(400 \mathrm{MHz}, \mathrm{CD}_{3} \mathrm{OD}\right): \delta 8.19(\mathrm{~s}, 1 \mathrm{H}), 7.77(\mathrm{~m}, 2 \mathrm{H}), 7.61(\mathrm{~m}$, 2H). MS (ESI) $224(\mathrm{M}+\mathrm{H})^{+}$.

(17) 4-(4-iodophenyl)-1H-1,2,3-triazole

White solid, $15 \%$ yield, ${ }^{1} \mathrm{H}-\mathrm{NMR}\left(400 \mathrm{MHz}, \mathrm{CD}_{3} \mathrm{OD}\right): \delta 8.19(\mathrm{~s}, 1 \mathrm{H}), 7.82(\mathrm{~d}, J=8.4 \mathrm{~Hz}, 2 \mathrm{H})$, $7.64(\mathrm{~d}, J=8.4 \mathrm{~Hz}, 2 \mathrm{H})$. MS (ESI) $272(\mathrm{M}+\mathrm{H})^{+}$.

(18) 4-(3-methylphenyl)-1H-1,2,3-triazole

White solid, $23 \%$ yield, ${ }^{1} \mathrm{H}-\mathrm{NMR}\left(400 \mathrm{MHz}, \mathrm{CD}_{3} \mathrm{OD}\right): \delta 8.14(\mathrm{~s}, 1 \mathrm{H}), 7.67(\mathrm{~s}, 1 \mathrm{H}), 7.62(\mathrm{~d}, J=7.7$

$\mathrm{Hz}, 1 \mathrm{H}), 7.34$ (t, J=7.6 Hz, 1H), 7.20 (d, J=7.6 Hz, 1H), 2.41 (s, 3H). MS (ESI) $160(\mathrm{M}+\mathrm{H})^{+}$.

(19) 3-(1H-1,2,3-triazol-4-yl)-phenol

White solid, $18 \%$ yield, ${ }^{1} \mathrm{H}-\mathrm{NMR}\left(400 \mathrm{MHz}, \mathrm{CD}_{3} \mathrm{OD}\right): \delta 8.09(\mathrm{~s}, 1 \mathrm{H}), 7.27(\mathrm{~m}, 3 \mathrm{H}), 6.81(\mathrm{~m}, 1 \mathrm{H})$. MS (ESI) $162(\mathrm{M}+\mathrm{H})^{+}$.

(20) 3-(1H-1,2,3-triazol-4-yl)-phenylamine

Tan solid, $19 \%$ yield, ${ }^{1} \mathrm{H}-\mathrm{NMR}\left(400 \mathrm{MHz}, \mathrm{CD}_{3} \mathrm{OD}\right): \delta 8.05(\mathrm{~s}, 1 \mathrm{H}), 7.12-7.20(\mathrm{~m}, 3 \mathrm{H}), 6.73-6.75$ $(\mathrm{m}, 1 \mathrm{H})$. MS (ESI) $161.2(\mathrm{M}+\mathrm{H})^{+}$.

(22) 4-(3-iodophenyl)-1H-1,2,3-triazole

White solid, $20 \%$ yield ${ }^{1} \mathrm{H}-\mathrm{NMR}\left(400 \mathrm{MHz}, \mathrm{CDCl}_{3}\right): \delta 8.21(\mathrm{~s}, 1 \mathrm{H}), 7.98(\mathrm{~s}, 1 \mathrm{H}), 7.81(\mathrm{~d}, J=7.8$ $\mathrm{Hz}, 1 \mathrm{H}), 7.73(\mathrm{~d}, J=8.1 \mathrm{~Hz}, 1 \mathrm{H}), 7.21(\mathrm{t}, J=7.8 \mathrm{~Hz}, 1 \mathrm{H})$. MS (ESI) $272(\mathrm{M}+\mathrm{H})^{+}$.

(23) 4-(3-bromophenyl)-1H-1,2,3-triazole

White solid, $2 \%$ yield, ${ }^{1} \mathrm{H}-\mathrm{NMR}\left(400 \mathrm{MHz}, \mathrm{CD}_{3} \mathrm{OD}\right): \delta 8.19(\mathrm{~s}, 1 \mathrm{H}), 8.05(\mathrm{~s}, 1 \mathrm{H}), 7.83(\mathrm{~d}, J=7.8$ $\mathrm{Hz}, 1 \mathrm{H}), 7.52(\mathrm{~d}, J=8.0 \mathrm{~Hz}, 1 \mathrm{H}), 7.37(\mathrm{t}, J=7.9 \mathrm{~Hz}, 1 \mathrm{H})$. MS (ESI) $224(\mathrm{M}+\mathrm{H})^{+}$.

(24) 4-(3,4-dibromophenyl)-1H-1,2,3-triazole

White solid, 23\% yield, ${ }^{1} \mathrm{H}-\mathrm{NMR}\left(400 \mathrm{MHz}, \mathrm{CD}_{3} \mathrm{OD}\right): \delta 8.25(\mathrm{~s}, 1 \mathrm{H}), 8.22(\mathrm{~s}, 1 \mathrm{H}), 7.76(\mathrm{~m}, 2 \mathrm{H})$. MS (ESI) $304(\mathrm{M}+\mathrm{H})^{+}$.

(25) 4-(1-benzofuran-2-yl)-1H-1,2,3-triazole

White solid, $25 \%$ yield, ${ }^{1} \mathrm{H}-\mathrm{NMR}\left(400 \mathrm{MHz}, \mathrm{CD}_{3} \mathrm{OD}\right): \delta 8.25(\mathrm{~s}, 1 \mathrm{H}), 7.65(\mathrm{~d}, J=7.6 \mathrm{~Hz}, 1 \mathrm{H})$, $7.56(\mathrm{~d}, J=8.0 \mathrm{~Hz}, 1 \mathrm{H}), 7.23-7.36(\mathrm{~m}, 3 \mathrm{H})$. MS (ESI) $186(\mathrm{M}+\mathrm{H})^{+}$.

(26) 2-(1H-1,2,3-triazol-4-yl)-pyridine

White solid, $57 \%$ yield, ${ }^{1} \mathrm{H}-\mathrm{NMR}\left(400 \mathrm{MHz}, \mathrm{CD}_{3} \mathrm{OD}\right): \delta 8.61(\mathrm{~m}, 1 \mathrm{H}), 8.32(\mathrm{~s}, 1 \mathrm{H}), 8.06(\mathrm{~d}$,

$J=8.0 \mathrm{~Hz}, 1 \mathrm{H}), 7.93(\mathrm{~m}, 1 \mathrm{H}), 7.40(\mathrm{~m}, 1 \mathrm{H}) . \mathrm{MS}(\mathrm{ESI}) 147(\mathrm{M}+\mathrm{H})^{+}$.

(27) 3-(1H-1,2,3-triazol-4-yl)-pyridine

White solid, $16 \%$ yield, ${ }^{1} \mathrm{H}-\mathrm{NMR}\left(400 \mathrm{MHz}, \mathrm{CD}_{3} \mathrm{OD}\right): \delta 9.06(\mathrm{~s}, 1 \mathrm{H}), 8.54(\mathrm{~d}, J=3.4 \mathrm{~Hz}, 1 \mathrm{H})$, $8.32(\mathrm{~m}, 2 \mathrm{H}), 7.55(\mathrm{~m}, 1 \mathrm{H})$. MS (ESI) $147(\mathrm{M}+\mathrm{H})^{+}$.

(28) 4-(1H-1,2,3-triazol-4-yl)-pyridine

White solid, $18 \%$ yield, ${ }^{1} \mathrm{H}-\mathrm{NMR}\left(400 \mathrm{MHz}, \mathrm{CD}_{3} \mathrm{OD}\right): \delta 8.51(\mathrm{~d}, J=4.7 \mathrm{~Hz}, 2 \mathrm{H}), 8.42(\mathrm{~s}, 1 \mathrm{H})$, $7.92(\mathrm{~d}, J=4.6 \mathrm{~Hz}, 2 \mathrm{H})$. MS (ESI) $147(\mathrm{M}+\mathrm{H})^{+}$.

(29) 2-(1H-1,2,3-triazol-4-yl)-3-methyl-pyridine

White solid, $35 \%$ yield, ${ }^{1} \mathrm{H}-\mathrm{NMR}\left(400 \mathrm{MHz}, \mathrm{CD}_{3} \mathrm{OD}\right): \delta 8.48(\mathrm{~d}, J=4.7 \mathrm{~Hz}, 1 \mathrm{H}), 8.20(\mathrm{~s}, 1 \mathrm{H})$, $7.79(\mathrm{~d}, J=7.6 \mathrm{~Hz}, 1 \mathrm{H}), 7.35(\mathrm{~m}, 1 \mathrm{H}), 3.32(\mathrm{~s}, 3 \mathrm{H})$. MS (ESI) $161(\mathrm{M}+\mathrm{H})^{+}$.

(30) 2-(1H-1,2,3-triazol-4-yl)-4-methyl-pyridine

White solid, $54 \%$ yield, ${ }^{1} \mathrm{H}-\mathrm{NMR}\left(400 \mathrm{MHz}, \mathrm{CD}_{3} \mathrm{OD}\right): \delta 8.45(\mathrm{~d}, J=5.1 \mathrm{~Hz}, 1 \mathrm{H}), 8.29(\mathrm{~s}, 1 \mathrm{H})$, $7.91(\mathrm{~s}, 1 \mathrm{H}), 7.23(\mathrm{~d}, J=5.1 \mathrm{~Hz}, 1 \mathrm{H}), 2.46(\mathrm{~s}, 3 \mathrm{H})$. MS (ESI) $161(\mathrm{M}+\mathrm{H})^{+}$.

(31) 2-(1H-1,2,3-triazol-4-yl)-5-methylpyridine

White solid, $28 \%$ yield, ${ }^{1} \mathrm{H}-\mathrm{NMR}\left(400 \mathrm{MHz}, \mathrm{CD}_{3} \mathrm{OD}\right): \delta 8.45(\mathrm{~s}, 1 \mathrm{H}), 8.27(\mathrm{~s}, 1 \mathrm{H}), 7.95(\mathrm{~d}, J=$ $8.1 \mathrm{~Hz}, 1 \mathrm{H}), 7.76(\mathrm{~d}, J=8.1 \mathrm{~Hz}, 1 \mathrm{H}), 2.41(\mathrm{~s}, 3 \mathrm{H})$. MS (ESI) $161(\mathrm{M}+\mathrm{H})^{+}$.

(32) 2-(1H-1,2,3-triazol-4-yl)-6-methylpyridine

Oil, $39 \%$ yield, ${ }^{1} \mathrm{H}-\mathrm{NMR}\left(400 \mathrm{MHz}, \mathrm{CD}_{3} \mathrm{OD}\right): \delta 8.33(\mathrm{~s}, 1 \mathrm{H}), 7.77-7.85(\mathrm{~m}, 2 \mathrm{H}), 7.26(\mathrm{~d}, J=7.4$ $\mathrm{Hz}, 1 \mathrm{H}), 2.60$ (s, 3H). MS (ESI) $161(\mathrm{M}+\mathrm{H})^{+}$.

4-(4-methoxyphenyl)-1H-1,2,3-triazole

White solid, $34 \%$ yield, ${ }^{1} \mathrm{H}-\mathrm{NMR}\left(400 \mathrm{MHz}, \mathrm{CDCl}_{3}\right): \delta 7.92(\mathrm{~s}, 1 \mathrm{H}), 7.76(\mathrm{~d}, J=8.8 \mathrm{~Hz}, 2 \mathrm{H}), 7.01$

(d, J=8.8 Hz, 2H), 3.88 (s, 3H). MS (ESI) $176(\mathrm{M}+\mathrm{H})^{+}$.

(13) 4-(1H-1,2,3-triazol-4-yl)-phenol 
To 4-(4-methoxyphenyl)-1 $H$-1,2,3-triazole ( $83 \mathrm{mg}, 0.5 \mathrm{mmol}$ ) was added hydrobromic acid (48\% in water, $2 \mathrm{~mL})$ and the solution was heated to $100^{\circ} \mathrm{C}$. After three hours, water $(10 \mathrm{~mL})$ and ethyl acetate $(10 \mathrm{~mL})$ were added. The organic products were collected in ethyl acetate, dried, filtered, and evaporated. The resulting residue was purified by preparative HPLC to afford the title compound as a white solid $\left(31 \mathrm{mg}, 40 \%\right.$ yield). ${ }^{1} \mathrm{H}-\mathrm{NMR}\left(400 \mathrm{MHz}, \mathrm{CD}_{3} \mathrm{OD}\right): \delta 8.01(\mathrm{~s}, 1 \mathrm{H})$, $7.65(\mathrm{~d}, J=8.7 \mathrm{~Hz}, 2 \mathrm{H}), 6.87(\mathrm{~d}, J=8.7 \mathrm{~Hz}, 2 \mathrm{H})$. MS (ESI) $162(\mathrm{M}+\mathrm{H})^{+}$.

N-(3-[1H-1,2,3-triazol-4-yl]phenyl)benzamide

White solid, $12 \%$ yield, ${ }^{1} \mathrm{H}-\mathrm{NMR}\left(400 \mathrm{MHz}, \mathrm{CD}_{3} \mathrm{OD}\right): \delta 8.19(\mathrm{~d}, J=10.3 \mathrm{~Hz}, 2 \mathrm{H}), 7.99(\mathrm{~d}, J=7.2$ $\mathrm{Hz}, 2 \mathrm{H}), 7.46-7.76(\mathrm{~m}, 6 \mathrm{H})$.

(21) Benzyl-(3-[1H-1,2,3-triazol-4-yl]phenyl)amine

To a cooled $\left(0^{\circ} \mathrm{C}\right)$ solution of $\mathrm{N}-(3-[1 \mathrm{H}-1,2,3$-triazol-4-yl]phenyl)benzamide (50 $\mathrm{mg}, 0.19 \mathrm{mmol})$ in THF $(0.5 \mathrm{~mL})$ and dioxane $(0.5 \mathrm{~mL})$ was added lithium aluminum hydride $(1.0 \mathrm{M}$ in THF, 0.2 $\mathrm{mL})$ and the reaction was allowed to warm to room temperature $16 \mathrm{~h}$. Additional dioxane (1 mL) and lithium aluminum hydride $(0.2 \mathrm{~mL})$ were added and the mixture was heated to $50^{\circ} \mathrm{C}$ for 30 min. Water and $\mathrm{Na}_{2} \mathrm{SO}_{4}$ were added and the residue was filtered. The filtrate was evaporated and purified by preparative HPLC to afford the title compound as a tan oil $\left(28 \mathrm{mg}, 60 \%\right.$ yield). ${ }^{1} \mathrm{H}-$ NMR (400MHz, CD 3 OD): $\delta 7.96(\mathrm{~s}, 1 \mathrm{H}), 7.40-7.43(\mathrm{~m}, 2 \mathrm{H})$ 7.30-7.35 (m, 2H), 7.04-7.25 (m, $4 \mathrm{H}), 6.63(\mathrm{~d}, J=8.0 \mathrm{~Hz}, 1 \mathrm{H}), 4.38(\mathrm{~s}, 2 \mathrm{H})$. MS (ESI) $251(\mathrm{M}+\mathrm{H})^{+}$.

(6) 4-(4-Methyl-phenyl)-1H-pyrazole

To a stirring solution of 2-(4-methylphenyl)-3-hydroxyacrolein $(0.20 \mathrm{~g}, 1.2 \mathrm{mmol})$ in ethanol (2.4 $\mathrm{mL})$ at $0^{\circ} \mathrm{C}$ was added hydrazine $(47 \mu \mathrm{L}, 1.5 \mathrm{mmol}) .{ }^{10}$ The reaction was allowed to warm to room temperature and was stirred overnight. Solid product formed which was removed by filtration, washed with ethanol, and dried to give the title compound as a yellow solid (87 mg, 46\%). ${ }^{1} \mathrm{H}-\mathrm{NMR}\left(400 \mathrm{MHz}, \mathrm{CD}_{3} \mathrm{OD}\right): \delta 7.96(\mathrm{~s}, 1 \mathrm{H}), 7.86(\mathrm{~s}, 1 \mathrm{H}), 7.46(\mathrm{~d}, J=8.0 \mathrm{~Hz}, 2 \mathrm{H}), 7.18$ $(\mathrm{d}, J=8.2 \mathrm{~Hz}, 2 \mathrm{H}), 2.34(\mathrm{~s}, 3 \mathrm{H})$. MS (ESI) $159(\mathrm{M}+\mathrm{H})^{+}$.

(7) 3-(4-Methyl-phenyl)-1H-pyrazole

To a stirring solution of 4-ethynyltoluene $(0.12 \mathrm{~g}, 1.0 \mathrm{mmol})$ in THF $(6 \mathrm{~mL})$ was added trimethylsilyl diazomethane $(2 \mathrm{M}$ in hexanes, $1.2 \mathrm{mmol}) .11$ The reaction was heated to $60^{\circ} \mathrm{C}$ for $72 \mathrm{~h}$. The solvent was evaporated and the residue was purified by preparative HPLC to afford the title compound as a yellow solid $\left(37 \mathrm{mg}, 23 \%\right.$ yield). ${ }^{1} \mathrm{H}-\mathrm{NMR}\left(400 \mathrm{MHz}, \mathrm{CD}_{3} \mathrm{OD}\right): \delta 7.63$ (m, $3 \mathrm{H}), 7.21(\mathrm{~d}, J=7.7 \mathrm{~Hz}, 2 \mathrm{H}), 6.60(\mathrm{~s}, 1 \mathrm{H}), 2.35(\mathrm{~s}, 3 \mathrm{H})$. MS (ESI) $159(\mathrm{M}+\mathrm{H})^{+}$.

\footnotetext{
${ }^{10}$ Kral, V.; Jelinek, L.; Saman, D. Preparation and synthetic utilization of diarylmethylenemalonaldehydes. Collect. Czech. Chem. Commun. 1989, 54, 2721-2730.

${ }^{11}$ Kirmse, W.; Horner, L. Reaction of phenylacetylene with azides and diazo compounds. Liebigs Ann. Chem. 1958, 614, 1-3.
} 
Elemental analysis results

\begin{tabular}{|c|c|c|c|c|c|c|c|}
\hline \\
\hline \multirow[b]{2}{*}{ Cpd. } & \multirow[b]{2}{*}{ mol. formula } & \multicolumn{3}{|c|}{ Calc. } & \multicolumn{3}{|c|}{ Found } \\
\hline & & $\mathbf{C}$ & $\mathbf{H}$ & $\mathbf{N}$ & $\mathbf{C}$ & $\mathbf{H}$ & $\mathbf{N}$ \\
\hline 3 & C9H9N3 & $67.91 \%$ & $5.70 \%$ & $26.40 \%$ & $67.78 \%$ & $5.69 \%$ & $26.12 \%$ \\
\hline 6 & C10H10N2 & $75.92 \%$ & $6.37 \%$ & $17.71 \%$ & $75.74 \%$ & $6.43 \%$ & $17.80 \%$ \\
\hline 7 & C10H10N2 & $75.92 \%$ & $6.37 \%$ & $17.71 \%$ & $75.65 \%$ & $5.96 \%$ & $17.61 \%$ \\
\hline 10 & C14H11N3 & $76.00 \%$ & $5.01 \%$ & $18.99 \%$ & $75.74 \%$ & $4.92 \%$ & $18.89 \%$ \\
\hline 12 & C11H13N3 & $70.56 \%$ & $7.00 \%$ & $22.44 \%$ & $70.44 \%$ & $6.97 \%$ & $22.44 \%$ \\
\hline 15 & C8H6ClN3 & $53.50 \%$ & $3.37 \%$ & $23.40 \%$ & $53.56 \%$ & $3.07 \%$ & $23.41 \%$ \\
\hline 16 & C8H6BrN3 & $42.88 \%$ & $2.70 \%$ & $18.75 \%$ & $42.83 \%$ & $2.78 \%$ & $18.51 \%$ \\
\hline 17 & C8H6IN3 & $35.45 \%$ & $2.23 \%$ & $15.50 \%$ & $35.55 \%$ & $2.06 \%$ & $15.34 \%$ \\
\hline 18 & C9H9N3 & $67.91 \%$ & $5.70 \%$ & $26.40 \%$ & $67.77 \%$ & $5.49 \%$ & $26.12 \%$ \\
\hline 24 & $\mathrm{C} 8 \mathrm{H} 5 \mathrm{Br} 2 \mathrm{~N} 3$ & $31.72 \%$ & $1.66 \%$ & $13.87 \%$ & $31.65 \%$ & $1.44 \%$ & $13.77 \%$ \\
\hline 26 & C7H6N4 & $57.53 \%$ & $4.14 \%$ & $38.34 \%$ & $57.26 \%$ & $4.05 \%$ & $38.00 \%$ \\
\hline 28 & C7H6N4 & $57.53 \%$ & $4.14 \%$ & $38.34 \%$ & $57.34 \%$ & $3.94 \%$ & $37.97 \%$ \\
\hline 30 & C8H8N4 & $59.99 \%$ & $5.03 \%$ & $34.98 \%$ & $59.91 \%$ & $4.77 \%$ & $34.82 \%$ \\
\hline 31 & C8H8N4 & $59.99 \%$ & $5.03 \%$ & $34.98 \%$ & $59.64 \%$ & $4.84 \%$ & $34.62 \%$ \\
\hline 32 & C8H8N4 & $59.99 \%$ & $5.03 \%$ & $34.98 \%$ & $59.72 \%$ & $4.92 \%$ & $34.71 \%$ \\
\hline
\end{tabular}

